\title{
VolumE maNagement Under body composition monitoring in critically ill patientS on CRRT: study protocol for a randomized controlled trial (VENUS trial)
}

Hyung Jung $\mathrm{Oh}^{1,2}$, Jung Nam An ${ }^{3,4}$, Sohee $\mathrm{Oh}^{5}$, Harin Rhee ${ }^{6,7}$, Jung Pyo Lee ${ }^{4,8}$, Dong Ki Kim ${ }^{8}$, Dong-Ryeol Ryu ${ }^{9,10}$ and Sejoong $\mathrm{Kim}^{11^{*}}$ (1)

\begin{abstract}
Background: Despite recent technical advances in the management of acute kidney injury (AKI), such as continuous renal replacement therapy (CRRT), intensive care unit mortality is still high, at approximately 40 to $50 \%$. Although several factors have been reported to predict mortality in AKI patients, fluid overload (FO) during CRRT is a well-known predictor of patient survival. However, FO has been mostly quantified as an arithmetical calculation and determined on the basis of the physicians' perception. Even though such quantification and assessment provides an easy evaluation of a patient's fluid status and is a simple method, it is not applicable unless a detailed record of fluid monitoring is available. Furthermore, the method cannot differentiate excess water in individual water compartments but can only reflect excess total body water. Bioimpedance analysis (BIA) has been used to measure the nutritional component of body composition and is a promising tool for the measurement of volume status. However, there has been no prospective interventional study for fluid balance among CRRT-treated AKI patients using BIA. Therefore, we will investigate the usefulness of fluid management using the InBody S10 (InBody ${ }^{\oplus}$, Seoul, Korea), a BIA tool, compared with that of generally used quantification methods.
\end{abstract}

Methods/design: This will be a multicenter, prospective, randomized controlled trial. A total of 244 patients undergoing CRRT treatment will be enrolled and randomly assigned to receive either to InBody S10-guided management or to fluid management based only on clinical information for 7 days. The primary outcome is to compare the rate of euvolemic status 7 days after the initiation of CRRT, with a secondary outcome being to compare the 28-, 60-, and 90-day mortality rates between the two groups.

Discussion: This will be the first clinical trial to investigate the effect of using BIA-guided fluid management to achieve euvolemia in CRRT-treated AKI patients.

Trial registration: ClinicalTrials.gov, ID: NCT03330626. Registered on 6 November 2017.

Keywords: Fluid balance, Bioimpedance analysis (InBody S10 (InBody $\left.{ }^{\oplus}\right)$ Seoul, Korea), Continuous renal replacement therapy (CRRT)

\footnotetext{
* Correspondence: sejoong@snubh.org

${ }^{11}$ Department of Internal Medicine, Seoul National University Bundang

Hospital, 82, Gumi-ro 173 beon-gil, Bundang-gu, Seongnam-si, Gyeonggi-do

13620, Republic of Korea

Full list of author information is available at the end of the article
}

(c) The Author(s). 2018 Open Access This article is distributed under the terms of the Creative Commons Attribution 4.0 International License (http://creativecommons.org/licenses/by/4.0/), which permits unrestricted use, distribution, and reproduction in any medium, provided you give appropriate credit to the original author(s) and the source, provide a link to the Creative Commons license, and indicate if changes were made. The Creative Commons Public Domain Dedication waiver (http://creativecommons.org/publicdomain/zero/1.0/) applies to the data made available in this article, unless otherwise stated. 


\section{Background}

Continuous renal replacement therapy (CRRT) has been established to manage the excretion of toxins and the balance of electrolytes and fluids in critically ill patients with acute kidney injury (AKI) [1-4]. Despite technical advances in the management of AKI over the past few years $[5,6]$, intensive care unit (ICU) mortality is still high at approximately 40 to $50 \%$ [7-10]. Although several factors have been reported to predict mortality in AKI patients [7, 8], fluid overload (FO) at the initial time of CRRT is a well-known predictor of patient survival [7, 11-16]. A few recent studies have examined the effect of fluid accumulation on mortality: Neyra et al. [14] showed that higher cumulative fluid balance during $72 \mathrm{~h}$ of ICU admission was independently associated with hospital mortality regardless of AKI or chronic kidney disease (CKD) presence; in the Randomized Evaluation of Normal vs. Augmented Level of Replacement Therapy (RENAL) study, a negative mean daily fluid balance was consistently related to improved clinical outcomes [15]; and Garzotto et al. [16] emphasized the association between the severity and speed of fluid accumulation and ICU mortality.

However, FO has been mostly quantified as an arithmetical calculation: the difference between the sum of daily fluid intake and total output adjusted by body weight and determined based on the physician's perception. Even though such quantification and assessment is an easy mechanism to evaluate a patient's fluid status and is a basic method, it is not applicable unless a detailed record of the fluid monitoring is available, and it cannot differentiate water excess in individual water compartments; instead, the method can only reflect excess total body water (TBW) [17].

Bioimpedance analysis (BIA) has been used to measure the nutritional part of body composition, such as fat mass or fat-free mass (FFM), in diverse conditions [18, 19]. However, it has been used as a promising tool for the measurement of volume status [20]. With the electrical properties of body tissues, multifrequency-BIA (MF-BIA) differentiates extracellular water (ECW) or intracellular water (ICW) from TBW using different frequencies: $0,1,5,50,100,200,500$ or $1000 \mathrm{kHz}[21,22]$. To date, several studies have demonstrated the accuracy and clinical usefulness of MF-BIA in chronic hemodialysis or peritoneal dialysis patients [23-27]. Moreover, in critically ill patients, MF-BIA has been useful in assessing volume status [28] and net fluid removal using CRRT to successfully reduce TBW, ECW, and ICW [29]. Rhee et al. [17] investigated the effect of MF-BIA-defined volume status on the mortality of critically ill patients with AKI; the authors demonstrated that MF-BIA-defined excess volume parameters adjusted for the square of the patient's height $\left(\mathrm{H}^{2}\right)$, such as TBW/ $\mathrm{H}^{2}$ and $\mathrm{ICW} / \mathrm{H}^{2}$, were independently associated with higher in-hospital mortality in male patients with AKI undergoing CRRT. However, there has been no prospective interventional study for fluid balance among CRRT-treated AKI patients using the abovementioned BIA. Therefore, in this study, we will investigate the usefulness of fluid management using InBody S10 (InBody ${ }^{\oplus}$, Seoul, Korea), a BIA tool, and compared with a generally used quantification method.

\section{Methods/design Hypothesis}

BIA-guided fluid management will reach an euvolemic status in patients who are treated with CRRT better than fluid management guided by a generally used quantification method, which is calculated by the difference between the sum of daily fluid intake and total output adjusted by body weight and determined based on the physician's perception. Moreover, the patients who achieve a euvolemic status 7 days after the initiation of CRRT will have better clinical outcomes based upon 28-, 60-, and 90-day mortality than those of patients who are still in a higher-volume status at the same time.

\section{Study design}

This will be a multicenter, prospective, parallel-group, open-label, randomized controlled trial. It is an investigator-initiated clinical trial. We have followed the Standard Protocol Items: Recommendations for Interventional Trials (SPIRIT) 2013 Statement which defines standard protocol items for clinical trials (Additional file 1). The overall study algorithm is depicted in Fig. 1 and the SPIRIT and study schedule are given in Fig. 2. After enrollment, InBody S10 (InBody ${ }^{\oplus}$, Seoul, Korea) will be used to measure fluid status at $0,1,2$, and 7 days after the initiation of the CRRT in InBody S10-based fluid management group, but at 0 and 7 days in fluid management guided by a generally used quantification method. Physicians will not know the results of the InBody S10 measurement. Instead, another investigator will measure the InBody S10 and record the results. In the treatment group, the fluid monitoring will be managed according to Table 1, while the fluid balancing of the control group will be conducted based on the clinical information obtained by the physician, such as body weight, hemodynamic stability and daily intake and output. Clinical information including hemodynamic monitoring and laboratory data will be collected on the same days, and we will investigate the 28-, 60-, and 90-day survival status.

\section{Study participants and measurements}

All patients will be selected from among patients who must be treated with CRRT in four tertiary hospitals in Korea (Seoul National University Bundang Hospital, 


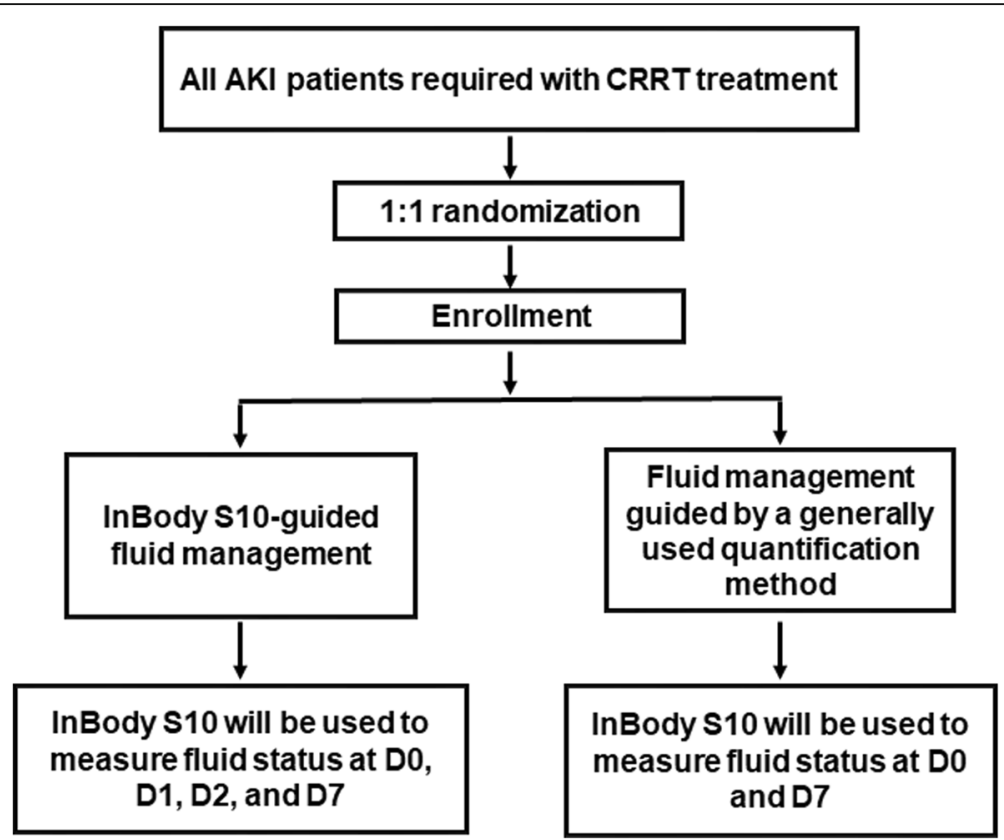

Fig. 1 The overall study algorithm. After enrollment, InBody S10 (InBody ${ }^{\oplus}$, Seoul, Korea) will be used to measure fluid status at 0, 1, 2, and 7 days after the initiation of the continuous renal replacement therapy (CRRT) in InBody S10-based fluid management group, but at 0 and 7 days in fluid management guided by a generally used quantification method. Abbreviations: AKI acute kidney injury, CRRT continuous renal replacement therapy, D0 the day of CRRT initiation, D1, 2 and 7 1,2 and 7 days from the day of CRRT initiation, respectively

\begin{tabular}{|c|c|c|c|c|c|c|c|c|c|}
\hline & \multirow[b]{2}{*}{$\begin{array}{l}\text { Enrolment } \\
\text { Allocation }\end{array}$} & \multicolumn{8}{|c|}{ STUDY PERIOD } \\
\hline & & \multicolumn{8}{|c|}{ Post-allocation } \\
\hline Timepoints (days) & D0 & $D 1$ & D2 & D3 & D4 & D5 & D6 & D7 & $E t c *$ \\
\hline \multicolumn{10}{|l|}{ Eligibility screen } \\
\hline \multirow{5}{*}{$\begin{array}{l}\text { Informed consent } \\
\text { Allocation } \\
\text { Demographics } \\
\text { Medical history } \\
\text { Fluid status measured by } \\
\text { InBody S10 }\end{array}$} & $\mathrm{X}$ & & & & & & & & \\
\hline & $\mathrm{X}$ & & & & & & & & \\
\hline & $\mathrm{X}$ & & & & & & & & \\
\hline & $\mathrm{X}$ & & & & & & & & \\
\hline & $\mathrm{X}$ & & & & & & & & \\
\hline \multicolumn{10}{|l|}{ Intervention } \\
\hline \multirow{3}{*}{$\begin{array}{l}\text { InBody S10-guided fluid } \\
\text { management } \\
\text { Control } \\
\text { Fluid management guided } \\
\text { by a generally used } \\
\text { quantification method }\end{array}$} & $\mathrm{X}$ & $\mathrm{X}$ & $\mathrm{X}$ & & & & & $\mathrm{X}$ & \\
\hline & & & & & & & & & \\
\hline & $\mathrm{X}$ & & & & & & & $\mathrm{X}$ & \\
\hline \multicolumn{10}{|l|}{ Assessment } \\
\hline \multirow{3}{*}{$\begin{array}{l}\text { Laboratory values } \\
T B W, I C W, E C W \text { at InBody } \\
\text { S10 } \\
\text { Fluid status }\end{array}$} & $\mathrm{X}$ & $\mathrm{X}$ & $\mathrm{X}$ & & & & & $\mathrm{X}$ & \\
\hline & $\mathrm{X}$ & $\mathrm{X}$ & $\mathrm{X}$ & & & & & $\mathrm{X}$ & \\
\hline & $\mathrm{X}$ & $\mathrm{X}$ & $\mathrm{X}$ & & & & & $\mathrm{X}$ & \\
\hline \multicolumn{10}{|l|}{ Outcomes } \\
\hline$T B W / H^{2}$ & & & & & & & & $\mathrm{X}$ & \\
\hline 28-, 60-, 90-day mortality & & & & & & & & & $\mathrm{X}$ \\
\hline
\end{tabular}

Fig. 2 Schedule of enrollment, interventions, and assessments according to the Standard Protocol Items: Recommendations for Interventional Trials (SPIRIT) guideline. Abbreviations: TBW total body water, ICW intracellular water, ECW extracellular water, TBW/H² total body water/height ${ }^{2}$ 
Table 1 The guideline of fluid management for treatment group

\begin{tabular}{ll}
\hline TBW/H $H^{2}$ at D0, D1, D2 & Target fluid removal amount $\left(L / H^{2} /\right.$ day $)$ \\
\hline $13-14$ & -0.5 \\
$14-15$ & -0.5 \\
$15-16$ & -0.6 \\
$16-17$ & -0.6 \\
$17-18$ & -0.7 \\
$18-19$ & -0.7 \\
$19-$ & -0.7 \\
\hline
\end{tabular}

Fluid removal will be determined based on the TBW/ $\mathrm{H}^{2}$ for 3 days (D0, D1, D2). However, fluid will be removed by $1 \mathrm{~L} /$ day if calculated fluid removal from the $\mathrm{TBW} / \mathrm{H}^{2}$ is over $1 \mathrm{~L} /$ day

Seoul National University Hospital, Seoul National University Boramae Medical Center, and Ewha Womans University Mokdong Hospital). Patients aged over 18 years who require CRRT for AKI will be screened for study participation according to the inclusion and exclusion criteria presented in Table 2.

\section{Randomization}

A research coordinator will perform the randomization. A list of random numbers will be generated by an independent statistician. Eligible participants will be randomly assigned 1:1 to either the control group (fluid management based on the clinical information alone) or the treatment group (InBody S10 (InBody, Seoul, Korea)-guided fluid management alone). Randomization will be stratified based on the institution and will utilize a randomized block design.

\section{Outcome measures}

The primary outcome is to compare the rate of euvolemic status at 7 days from the CRRT start. The euvolemic status will be considered different when $\mathrm{TBW} / \mathrm{H}^{2}$ between the day of CRRT initiation (D0) and 7 days from the day of CRRT initiation (D7) is less than -2.1 or $\mathrm{TBW} / \mathrm{H}^{2}$ at $\mathrm{D} 7$ is less than $13 \mathrm{~L} / \mathrm{m}^{2}$. According to Rhee's recent study, the group reaching $\mathrm{TBW} / \mathrm{H}^{2}<13$ $\mathrm{L} / \mathrm{m}^{2}$ had a significantly enhanced survival rate compared with that of the group that could not reach $\mathrm{TBW} / \mathrm{H}^{2}<13 \mathrm{~L} / \mathrm{m}^{2}$ [17]. Moreover, our further study has revealed that the survival rate was significantly increased when the difference in $\mathrm{TBW} / \mathrm{H}^{2}$ between CRRT initiation and at 7 days from CRRT start $\left(\mathrm{TBW} / \mathrm{H}^{2}\right.$ at 7 days $-\mathrm{TBW} / \mathrm{H}^{2}$ at CRRT initiation) was less than $-2.1 \mathrm{~L} / \mathrm{m}^{2}$, even though it was not sufficient to reach $\mathrm{TBW} / \mathrm{H}^{2}<13 \mathrm{~L} / \mathrm{m}^{2}$ (data not shown). Therefore, we will also consider the euvolemia as $\mathrm{TBW} / \mathrm{H}^{2}$ at 7days - TBW $/ \mathrm{H}^{2}$ at CRRT initiation $<-2.1 \mathrm{~L} / \mathrm{m}^{2}$ in this study. The secondary outcome is to investigate the 28-, 60-, and 90-day mortality rates in the control and treatment groups and to compare the mortality rates between the groups that reach euvolemic status or not for sub-analysis.

\section{Assessment of the fluid status}

For participants in the control group, clinical information obtained by the physician is the standard of judgment. However, the participants will be measured by InBody S10 (InBody $\left.{ }^{\circ}\right)$ at D0 and D7, and the results will

Table 2 Inclusion and exclusion criteria

Inclusion criteria

Patients will be eligible for inclusion in the study if all the following criteria are met:

1. The treating clinician believes that the patient requires continuous renal replacement therapy for acute kidney injury

2. The treating clinicians anticipate treating the patient with continuous renal replacement therapy for at least $72 \mathrm{~h}$

3. Informed consent has been obtained

4. The patient fulfills one of the following clinical criteria for initiating continuous renal replacement therapy:

Urine output $<100 \mathrm{~mL} / 6 \mathrm{~h}$ that has been unresponsive to fluid resuscitation measures

$\mathrm{K}^{+}>6.5 \mathrm{mmol} / \mathrm{L}$

$\mathrm{pH}<7.2$

Urea $>25 \mathrm{mmol} / \mathrm{L}$

Clinically significant organ edema in the setting of acute kidney injury

5. Patients who are over $5 \%$ of fluid overload or their total body water/height ${ }^{2} \geq 13 \mathrm{~L} / \mathrm{m}^{2}$

Exclusion criteria

Patients will be excluded from the study if, in the opinion or knowledge of the responsible clinician any of the following criteria are present:

1. Patient age is $<18$ years

2. Death is imminent $(<24 \mathrm{~h})$

3. There is a strong likelihood that the study treatment will not be continued in accordance with the study protocol.

4. The patient has been treated with continuous renal replacement therapy or other dialysis previously during the same hospital admission.

5. The patient has been on maintenance dialysis prior to the current hospitalization.

6. Any other major illness that, in the investigator's judgment, will substantially increase the risk associated with the subject's participation in this study. 
be recorded. Clinical information will be composed of four items: body weight in the morning; hemodynamic stability; absence of symptoms and signs of hypervolemia (dyspnea, edema, and crackle) or hypovolemia (dizziness and orthostatic hypotension); and daily intake and output. For participants in the treatment group, InBody S10 (InBody ${ }^{\circ}$ ) will also be measured at 9:00 a.m. on D0, D1, D2, and D7. Table 1 shows the guideline of fluid management for the treatment group. However, both the physicians and participants will be blinded to the results of InBody S10 (InBody ${ }^{\circ}$ ) analysis.

InBody $\mathrm{S} 10\left(\right.$ InBody $\left.^{\circ}\right)$ is a medical device that has been approved by the US Food and Drug Administration as an impedance body-fat analyzer. The device provides the following parameters: TBW, ICW, ECW, segmental water values, ECW ratio (ECW/TBW), FFM, soft lean mass (SLM), segmental lean mass, body fat mass (BFM), percentage body fat (PBF), and TBW/FFM.

The BIA method calculates body composition using the resistance value (impedance) that appears due to the difference in electrical conductivity according to the biological characteristics of each tissue. Electrical conductivity is proportional to the amount of water and electrolytes, and the water content of fat tissue is relatively smaller than that of other tissues, which leads to a decrease in electrical conductivity when the fat content increases. Among the components of the body, body water is the component that passes an electrical current, so the volume of water in the body can be obtained by measuring the resistance value obtained from body water [21].

\section{Clinical and laboratory evaluations}

Physical examination, comorbidity and medication will be reviewed. Laboratory evaluations, including complete blood cell counts $(\mathrm{CBC})$, electrolytes, creatinine, protein, albumin, calcium, phosphorous, total cholesterol, triglyceride, low-density lipoprotein cholesterol, high-sensitivity $\mathrm{C}$-reactive protein (hs-CRP), and $\mathrm{N}$-terminal prohormone of brain natriuretic peptide (NT-proBNP) will also be performed in the morning in the intensive care unit.

\section{Safety issues}

The InBody S10 (InBody ${ }^{\circ}$ method approved by the US Food and Drug Administration has been used in clinical practice. Applying weak alternating currents into the body is known to not be harmful.

\section{Sample size calculations}

No previous report has evaluated the effect of InBody S10 $\left(\right.$ InBody $\left.{ }^{\circ}\right)$-guided fluid management on CRRT-treated patients. We will randomly divide the enrolled participants equally into two groups. Assuming a $19 \%$ difference in the rate of achieving euvolemic status between the two groups with 0.8 of power, two-sided, and 0.05 of alpha, 97 participants will be allocated in each group. However, 122 participants will be required to enroll in each group due to a $20 \%$ withdrawal rate.

\section{Statistical analyses}

The statistical analyses will be conducted both on a per-protocol (PP) and an intention-to treat (ITT) basis. For PP analysis, all participants who complete the study will be included to evaluate the primary and secondary outcomes. For the ITT analysis, all participants who are enrolled and randomized to one of the two groups and who complete the first visit will be included. Basic statistics will be reported in terms of the mean \pm SD for continuous variables, or as percentages for categorical variables. Differences between groups will be analyzed using Student's $t$ test for continuous variables and the $\chi^{2}$ test or Fisher's exact test for categorical variables. The primary outcome will be compared with Student's $t$ test. An analysis of covariance (ANCOVA) will be used to analyze the primary outcome as the secondary analysis to adjust the baseline value. Multivariate Cox proportional hazard regression models will be used to analyze the time to mortality. Although the institutions are mainly located in large cities, thereby limiting the likelihood of any important cluster effect, we will be sure to accommodate possible clustering in our models and analysis as required. A value of $P<0.05$ will be considered statistically significant. All analyses will be performed using SPSS Statistics software (v21.0; IBM Corporation, Armonk, NY, USA).

\section{Ethics approval}

The study will be performed in accordance with the Declaration of Helsinki, as amended by the 59th World Medical Association General Assembly in 2008. All the participants will provide signed, informed, written consent, stating that participation is voluntary and can be withdrawn at any time. Approval for the study has been obtained from the Institutional Review Board of Seoul National University Bundang Hospital (B-1702/383-003), Seoul National University Hospital (J-1705-080-855), Seoul National University Boramae Medical Center (20,170,516/20-2017-2/062), Ewha Womans University Mokdong Hospital (EUMC 2017-05-049-008) (Ethical Approval Document). The trial protocol has been registered at https://clinicaltrials.gov/ct2/show/NCT03330626 (NCT03330626). Moreover, this research was supported by a grant from the Korea Health Technology R\&D Project through the Korea Health Industry Development Institute (KHIDI), funded by the Ministry of Health and Welfare, Republic of Korea (grant number: HI17C1827) (Funding Documentation). 


\section{Discussion}

CRRT is a generally used tool to manage fluid balance among the critically ill patients with AKI when they have medically refractory overhydration [1-4]. Moreover, some recent studies have emphasized the effects of cumulative fluid balance on mortality among CRRT-treated AKI patients [14-16]. However, FO has been mostly quantified as an arithmetical calculation using the following equation: (The sum of daily fluid intake - total output) / (Body weight). This condition has been determined based on the physician's perception. However, it is not applicable unless a detailed record of fluid monitoring is available, and it cannot differentiate water excess in individual water compartments [17], although such quantification and assessment represents an easy and basic method to evaluate a patient's fluid status.

InBody $\mathrm{S} 10$ (InBody ${ }^{\odot}$ ) is a medical device using MF-BIA and has been approved by the US Food and Drug Administration as an impedance body-fat analyzer that also provides analysis of individual water compartments, such as TBW, ICW, ECW, segmental water values, and ECW ratio (ECW/TBW).

MF-BIA has proven to be useful for assessing volume status in critically ill patients [28, 29] and Rhee et al. [17] showed the effect of MF-BIA-defined volume status on the mortality of critically ill patients with AKI using InBody S20 $\left(\operatorname{InBody}^{\circ}\right)$. As there has not yet been a prospective interventional study of fluid balance among CRRT-treated AKI patients using the abovementioned BIA, we will investigate the usefulness of fluid management using the BIA device InBody S10 (InBody ${ }^{\circ}$ ) and compare the results with a generally used quantification method.

To our knowledge, this will be the first multicenter, prospective, randomized controlled trial to assess whether InBody S10-guided volume management is better able to reach a euvolemic status at 7 days after the initiation of CRRT than the quantitation method for volume management is. The endpoint of the VENUS (VolumE maNagement Under body composition monitoring in critically ill patientS on CRRT) study may be of the utmost importance to healthcare providers.

So far, there is no definite index for determining euvolemic status. Instead, most physicians determine their patients' volume status based on clinical information, such as intake and output, chest radiographic examination, and physical examination. Therefore, the fluid balance of patients may be managed differently based on the physicians' opinions, even with the same patients. The abovementioned study by Rhee et al. [17] suggested that $\mathrm{TBW} / \mathrm{H}^{2}$ and $\mathrm{ICW} / \mathrm{H}^{2}$ were independently associated with higher in-hospital mortality in male patients with AKI undergoing CRRT, and the group reaching $\mathrm{TBW} / \mathrm{H}^{2}<13 \mathrm{~L} / \mathrm{m}^{2}$, which was $13 \%$ of total enrolled patients, had a significantly increased level of survival compared to that of other patients. Thus, we will define the euvolemia as the status of $\mathrm{TBW} / \mathrm{H}^{2}<13 \mathrm{~L} / \mathrm{m}^{2}$. In addition, our study of fluid removal has revealed that the difference in $\mathrm{TBW} / \mathrm{H}^{2}$ between CRRT initiation and at day 7 of CRRT, $\left[\left(B W / H^{2}\right.\right.$ at 7 days $-\mathrm{TBW} / \mathrm{H}^{2}$ at CRRT initiation), especially when it was greater than $-2.1 \mathrm{~L} / \mathrm{m}^{2}$, was significantly associated with an increased mortality rate, even though it did not reach $\mathrm{TBW} / \mathrm{H}^{2}<13 \mathrm{~L} / \mathrm{m}^{2}$ (data not shown). Therefore, we will also consider another measure of euvolemia in this study, namely $\mathrm{TBW} / \mathrm{H}^{2}$ at 7 days $-\mathrm{TBW} / \mathrm{H}^{2}$ at CRRT initiation $<-2.1 \mathrm{~L} / \mathrm{m}^{2}$.

There is a chance that patients within the same institution will be correlated more closely than patients at different institutions will. To avoid this issue, we will investigate for the possible clustering of patients at the recruitment institution level and will accommodate such clustering in our models and analysis as required. We have also conservatively calculated the sample size, assuming a high drop-out rate, to overcome this potential problem.

There are a few limitations in this study. First, this study will be investigated in Korean Tertiary Hospitals, which means that the results will require cautious interpretation concerning ethnicity. Second, we have defined euvolemia arbitrarily, although this definition was based on recent data and upon our own study. However, this study is the first study, to the best our knowledge, to determine guidelines for the fluid balance in CRRT-treated critically ill AKI patients using a prospective randomized controlled trial. We expect that the endpoint of the VENUS study will provide the utmost information to healthcare providers.

In summary, the VENUS study is the first prospective, randomized controlled trial to evaluate the clinical usefulness of InBody S10-guided fluid management in CRRT-treated critically ill AKI patients. The aim of this study is to identify more objective guidelines to manage volume status in CRRT-treated, critically ill AKI patients.

\section{Trial status}

This trial is ongoing. Participants are currently being recruited.

\section{Additional file}

Additional file 1: Standard Protocol Items: Recommendations for Interventional Trials (SPIRIT) 2013 Checklist: recommended items to address in a clinical trial protocol and related documents. (DOC $124 \mathrm{~kb}$ )

\section{Acknowledgements}

Not applicable.

\section{Funding}

This research was supported by a grant from the Korea Health Technology R\&D Project through the Korea Health Industry Development Institute (KHIDI), funded by the Ministry of Health and Welfare, Republic of Korea (grant number: HI17C1827) (Funding Documentation). 


\section{Availability of data and materials}

Not applicable.

\section{Authors' contributions}

HJO and SK drafted the manuscript. SO, HR, JPL, DKK, and DRR participated in the design of the study and the data collection. JNA, JPL, DKK, and DRR participated in the design of the study, drafted the manuscript, conceived the study, and participated in acquiring funding. SK had final responsibility for the decision to submit this manuscript for publication. All authors read and approved the final submitted manuscript.

\section{Ethics approval and consent to participate}

The study will be performed in accordance with the Declaration of Helsinki, as amended by the 59th World Medical Association General Assembly in 2008. All the participants will provide signed, informed, written consent, stating that participation is voluntary and can be withdrawn at any time. Approval for the study has been obtained from the Institutional Review Board of Seoul National University Bundang Hospital (B-1702/383-003), Seoul National University Hospital (J-1705-080-855), Seoul National University Boramae Medical Center (20,170,516/20-2017-2/062), Ewha Womans University Mokdong Hospital (EUMC 2017-05-049-008). The trial protocol has been registered at http://www.clinicaltrials.gov (NCT03330626) (Ethical Approval Document).

\section{Consent for publication}

Not applicable.

\section{Competing interests}

The authors declare that they have no competing interests.

\section{Publisher's Note}

Springer Nature remains neutral with regard to jurisdictional claims in published maps and institutional affiliations.

\begin{abstract}
Author details
'Ewha Institute of Convergence Medicine, Ewha Womans University Mokdong Hospital, Seoul, Republic of Korea. ${ }^{2}$ Research Institute for Human Health Information, Ewha Womans University Mokdong Hospital, Seoul, Republic of Korea. ${ }^{3}$ Department of Critical Care Medicine, Seoul National University Boramae, Medical Center, Seoul, Republic of Korea. ${ }^{4}$ Department of Internal Medicine, Seoul National University Boramae, Medical Center, Seoul, Republic of Korea. ${ }^{5}$ Department of Biostatistics, Seoul National University Boramae, Medical Center, Seoul, Republic of Korea. ${ }^{6}$ Department of Internal Medicine, Pusan National University School of Medicine, Busan, Republic of Korea. ${ }^{7}$ Biomedical Research Institute, Pusan National University Hospital, Busan, Republic of Korea. ${ }^{8}$ Department of Internal Medicine, Seoul National University College of Medicine, Seoul, Republic of Korea. ${ }^{9}$ Department of Internal Medicine, School of Medicine, Ewha Womans University, Seoul, Republic of Korea. ${ }^{10}$ Tissue Injury Defense Research Center, Ewha Womans University, Seoul, Republic of Korea. ${ }^{11}$ Department of Internal Medicine, Seoul National University Bundang Hospital, 82, Gumi-ro 173 beon-gil, Bundang-gu, Seongnam-si, Gyeonggi-do 13620, Republic of Korea.
\end{abstract}

Received: 19 July 2018 Accepted: 15 November 2018 Published online: 12 December 2018

\section{References}

1. Ostermann $M$, Joannidis $M$, Pani $A$, et al. Patient selection and timing of continuous renal replacement therapy. Blood Purif. 2016;42:224-37.

2. Bagshaw SM, Chakravarthi MR, Ricci Z, et al. Precision continuous rena replacement therapy and solute control. Blood Purif. 2016;42:238-47.

3. Murugan $\mathrm{R}$, Hoste $\mathrm{E}$, Mehta RL, et al. Precision fluid management in continuous renal replacement therapy. Blood Purif. 2016;42:266-78.

4. Cerda J, Baldwin I, Honore PM, Villa G, Kellum JA, Ronco C. Role of technology for the management of AKI in critically ill patients: from adoptive technology to precision continuous renal replacement rherapy. Blood Purif. 2016:42:248-65.

5. Ronco C, Bellomo R, Homel P, et al. Effects of different doses in continuous veno-venous haemofiltration on outcomes of acute renal failure: a prospective randomised trial. Lancet. 2000;356:26-30.
6. Heering P, Morgera S, Schmitz FJ, et al. Cytokine removal and cardiovascular hemodynamics in septic patients with continuous venovenous hemofiltration. Intensive Care Med. 1997;23:288-96.

7. Uchino S, Kellum JA, Bellomo R, et al. Acute renal failure in critically ill patients: a multinational, multicenter study. JAMA. 2005;294:813-8.

8. Nisula S, Kaukonen KM, Vaara ST, et al. Incidence, risk factors and 90-day mortality of patients with acute kidney injury in Finnish intensive care units: the FINNAKI study. Intensive Care Med. 2013;39:420-8.

9. Oh HJ, Kim MH, Ahn JY, et al. Can early initiation of continuous renal replacement therapy improve patient survival with septic acute kidney injury when enrolled in early goal-directed therapy? J Crit Care. 2016;35:51-6.

10. Oh HJ, Shin DH, Lee MJ, et al. Early initiation of continuous renal replacement therapy improves patient survival in severe progressive septic acute kidney injury. J Crit Care. 2012;27:743.e9-18.

11. Bagshaw SM, Brophy PD, Cruz D, Ronco C. Fluid balance as a biomarker: impact of fluid overload on outcome in critically ill patients with acute kidney injury. Crit Care. 2008;12:169.

12. Cerda J, Sheinfeld G, Ronco C. Fluid overload in critically ill patients with acute kidney injury. Blood Purif. 2010;29:331-8.

13. Metnitz PG, Krenn CG, Steltzer $H$, et al. Effect of acute renal failure requiring renal replacement therapy on outcome in critically ill patients. Crit Care Med. 2002;30:2051-8.

14. Neyra JA, Li X, Canepa-Escaro F, et al. Cumulative fluid balance and mortality in septic patients with or without acute kidney injury and chronic kidney disease. Crit Care Med. 2016;44:1891-900.

15. Bellomo R, Cass A, Cole L, et al. An observational study fluid balance and patient outcomes in the Randomized Evaluation of Normal vs. Augmented Level of Replacement Therapy trial. Crit Care Med. 2012;40:1753-60.

16. Garzotto F, Ostermann M, Martin-Langerwerf D, et al. The Dose Response Multicentre Investigation on Fluid Assessment (DoReMIFA) in critically ill patients. Crit Care. 2016;20:196

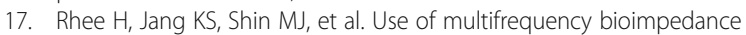
analysis in male patients with acute kidney injury who are undergoing continuous veno-venous hemodiafiltration. PLoS One. 2015;10:e0133199.

18. Kyle UG, Unger P, Dupertuis YM, Karsegard VL, Genton L, Pichard C. Body composition in 995 acutely ill or chronically ill patients at hospital admission: a controlled population study. J Am Diet Assoc. 2002:102:944-55.

19. Kyle UG, Bosaeus I, De Lorenzo AD, et al. Bioelectrical impedance analysispart II: utilization in clinical practice. Clin Nutr. 2004;23:1430-53.

20. Malbrain ML, Huygh J, Dabrowski W, De Waele JJ, Staelens A, Wauters J. The use of bio-electrical impedance analysis (BIA) to guide fluid management, resuscitation and deresuscitation in critically ill patients: a bench-to-bedside review. Anaesthesiol Intensive Ther. 2014;46:381-91.

21. Kyle UG, Bosaeus I, De Lorenzo AD, et al. Bioelectrical impedance analysis - part I: review of principles and methods. Clin Nutr. 2004;23:1226-43.

22. Chamney PW, Wabel P, Moissl UM, et al. A whole-body model to distinguish excess fluid from the hydration of major body tissues. Am J Clin Nutr. 2007:85:80-9.

23. O'Lone EL, Visser A, Finney H, Fan SL. Clinical significance of multi-frequency bioimpedance spectroscopy in peritoneal dialysis patients: independent predictor of patient survival. Nephrol Dial Transplant. 2014;29:1430-7.

24. Koh KH, Wong HS, Go KW, Morad Z. Normalized bioimpedance indices are better predictors of outcome in peritoneal dialysis patients. Perit Dial Int. 2011:31:574-82.

25. Alijanian N, Naini AE, Shahidi S, Liaghat L, Samani RR. The comparative evaluation of patients' body dry weight under hemodialysis using two methods: bioelectrical impedance analysis and conventional method. J Res Med Sci. 2012;17:923-7.

26. Tattersall J. Bioimpedance analysis in dialysis: state of the art and what we can expect. Blood Purif. 2009;27:70-4.

27. Kotanko P, Levin NW, Zhu F. Current state of bioimpedance technologies in dialysis. Nephrol Dial Transplant. 2008;23:808-12.

28. Basso F, Berdin G, Virzi GM, et al. Fluid management in the intensive care unit: bioelectrical impedance vector analysis as a tool to assess hydration status and optimal fluid balance in critically ill patients. Blood Purif. 2013;36:192-9.

29. Dabrowski W, Kotlinska-Hasiec E, Schneditz D, et al. Continuous venovenous hemofiltration to adjust fluid volume excess in septic shock patients reduces intra-abdominal pressure. Clin Nephrol. 2014:82:41-50. 